\author{
Andrea Giorgetti \\ Maria Vittoria Calloni \\ Roberto Freschi \\ Giuseppe Mariani \\ Patrizia Perrone \\ Patrizia Secchi \\ Adriana Sermoni
}

Received: 19 October 2000

Accepted in revised form: 9 January 2001

\section{Cerebral venous thrombosis after lumbar puncture and high steroid cycle in a patient with multiple sclerosis}

\begin{abstract}
The present case report underlies the importance of modifications in the characteristics of postlumbar puncture headache, which led us to diagnose cerebral venous thrombosis in a 34-year-old woman affected by multiple sclerosis. The patient underwent a lumbar puncture for diagnostic purposes and successively was treated with megadoses of methylprednisolone (1 g intravenously for 6 days). After lumbar puncture, the patient developed an orthostatic headache due to cerebrospinal fluid (CSF) hypotension. Eight days later, and two days after finishing the cycle of methylprednisolone, the headache worsened, became diffuse and persisting, and was longer modified by postural changes. This was associat-
\end{abstract}

ed with psychomotor slowing and, the day after, a partial epileptic status. Magnetic resonance imaging showed an alteration in the signal corresponding to the sagittal superior, transverse and sigmoid sinuses, indicative of venous thrombosis. The potential role of lumbar puncture, steroid treatment and other associated risk factors, and the importance of considering modifications in postpuncture headache as a condition which needs specific neuroradiological examinations are discussed.

Key words Cerebral venous thrombosis $\cdot$ Lumbar puncture $\cdot$ Headache

\section{Introduction}

Cerebral venous thrombosis (CVT) is an infrequent pathological condition that is sometimes unrecognized or diagnosed too late, in part because of the wide variability in clinical presentations. Although CVT occurs with a large spectrum of signs and symptoms, headache undoubtedly is the most frequent symptom, even in the absence of typical clinical characteristics and specific temporal profile [1]. Several potential causes and risk factors have been identified for CVT, including cranial trauma, hematological, metabolic or neoplastic diseases, pregnancy and puerperium, oral contraceptives and congenital abnormalities of thrombophilic status [2]. A few cases have been reported after lumbar punc- ture, myelography and, only rarely, after a lumbar puncture for diagnostic purposes [3, 4]. We describe a patient who developed CVT after a lumbar puncture for the diagnosis of multiple sclerosis and subsequent treatment with high doses of methyprednisolone.

\section{Case report}

The patient is a 34-year-old woman affected by migraine without aura and using estroprogestins as contraceptives. She is a non-smoker, and has no hypertension nor personal or family history of venous or arterial thrombosis. Approximately one year ago, the patient began to complain 
of paresthesiae of the fingers of the right hand, which sometimes irradiated to the right forearm. In the last 6 months, she reported visual dimming, dizziness and tendency to heel over to the right, associated with heaviness and awkward movements of the lower limbs. In the last few months she had difficulties in urination.

The neurological examination showed: inexhaustible horizontal-rotatory nystagmus in the gaze toward the left and upper sides; anisocoria right > left; non-sensorimotor deficits of the upper and lower limbs; quick, polykinetic osteotendinous reflexes at the lower limbs with knee and feet clonus; bilaterally positive Hoffmann's sign; plantar cutaneous stimulus bilaterally incorrect; absent superficial abdominal reflexes; some uncertainty at the nose-index finger test; and lateropulsion to the right at Romberg's maneuver.

Magnetic resonance imaging (MRI) of the brain showed multiple, round demyelinating lesions, partly confluent, in the white matter of both cerebral and cerebellar hemispheres
(Fig. 1a). Multimodal evoked potentials were also altered. The cerebrospinal fluid (CSF) examination showed a slight increase in protein content $(69 \mathrm{mg} / \mathrm{dl})$ and several oligoclonal bands were revealed by isoelectrofocusing. A treatment with methyprednisolone was therefore undertaken with a dosage of $1 \mathrm{~g}$ intravenously for 6 days.

In the two days following lumbar puncture, the patient developed a slight, bilateral, diffuse headache, which worsened when she assumed an orthostatic position and was relieved by a clinostatic position. Eight days after lumbar puncture and therefore two days after completion of the steroid treatment, the headache reappeared with characteristics completely different from those of the previous headache. It was gravative, diffuse and persisting for the entire day, was not modified by the posture assumed and was associated with psychomotor slowing. Moreover, clonic spasm appeared at the right hand, without corresponding epileptic features on electroencephalography
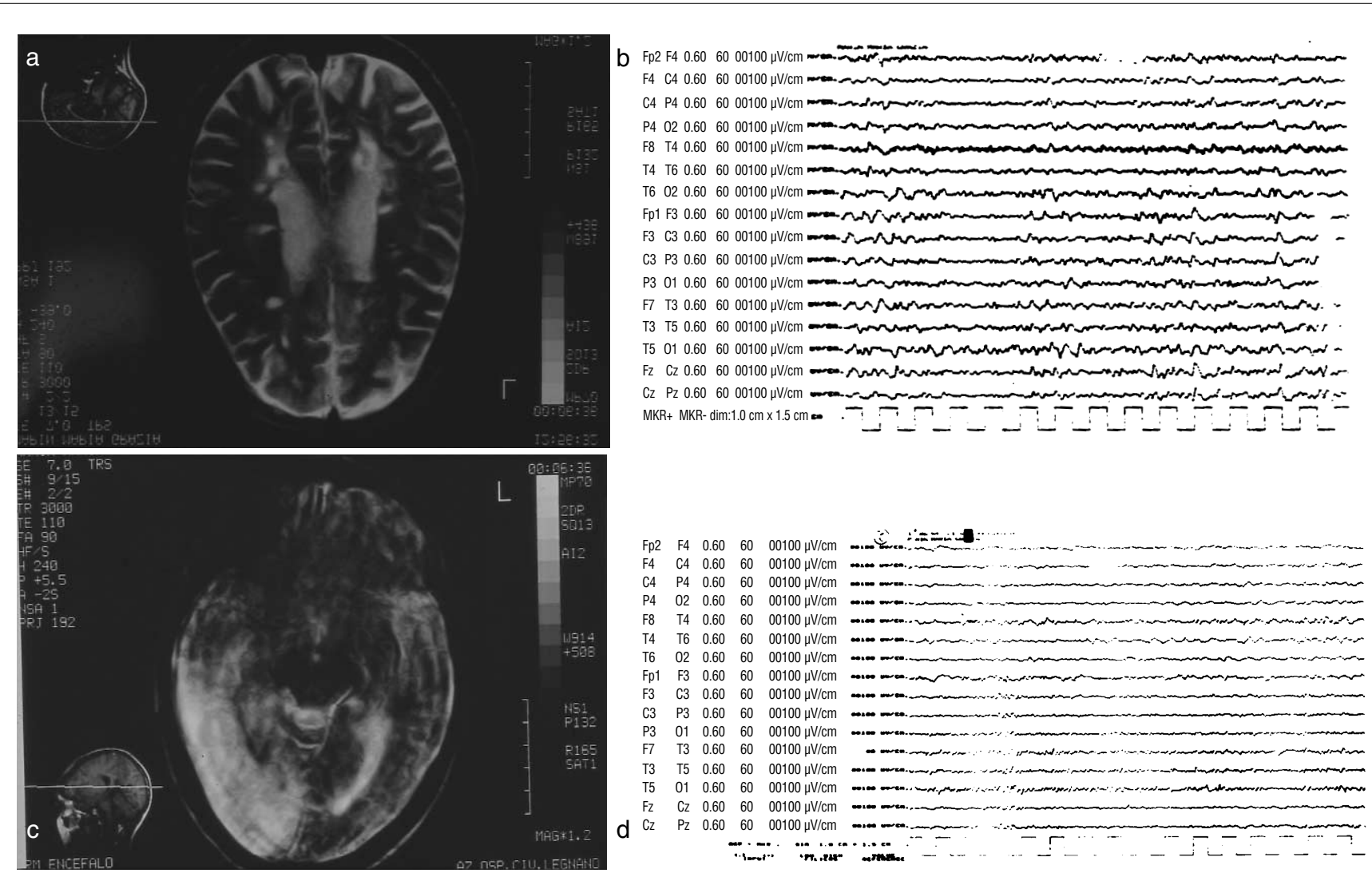

Fig. 1 a Multiple nodular lesions in cerebral and cerebellar hemispheres, compatible with demyelinating lesions (first MRI examination; long TR sequence). b Low left hemispheric activity, without epileptic abnormalities (first EEG examination). $\mathbf{c}$ Large hyperintense area in the right temporo-occipital region, suggestive of an ischemic lesion (second MRI examination; long TR sequence). d Slow cuspidate abnormalities in the left temporal region and slow diffuse activity in the right hemisphere (second EEG examination) 
(EEG), which, on the other hand, showed a slow left hemispheric activity with diffusion and slowing of the background rhythm (Fig. 1b). One day later, a partial epileptic status developed (with tonic-clonic crises, succeeding in the right hemibody) which regressed after intravenous administration of phenytoin soluble ( $1 \mathrm{~g}$ in $100 \mathrm{ml}$ saline). Computed tomography (CT) of the brain showed a right temporo-occipital hypodensity with a modest haemorrhagic component along the course of the transverse sinus, suggesting venous thrombosis. MRI confirmed this hypothesis, and showed a right temporo-occipital ischemic area along with thrombosis of transverse, sigmoid and superior sagittal sinuses (Fig. 1c). Subsequent EEG examinations showed persisting slow cuspidate abnormalities in the left temporal area and a slowing of the background activity in the right hemisphere (Fig. 1d). Fundus oculi examination showed an initial bilateral papilledema.

Hematochemical, rheumatological and immunological parameters were all in the normal range. Thrombophilic screening did not reveal any abnormality. In particular, antiphospholipid antibodies were absent and the level of resistance-activated protein $\mathrm{C}$ was normal.

The patient was treated with anticoagulants (starting with an intravenous bolus of heparin followed by a continuous infusion with a pump according to activated partial thromboplastin time (aPTT) for 5 days and successively with dicumarole per os, maintaining international normalized ratio (INR) values between 2 and 3 ) and with anti-epileptic drugs (diphenylhyantoin, $200 \mathrm{mg} /$ day). Progressive neurological recovery was observed. At discharge, a left homonymous lateral hemianopsia persisted whereas the other signs present at admission were unchanged.

\section{Discussion}

The occurrence of CVT during treatment with high dosages of methylprednisolone and after lumbar puncture for diagnostic purposes leads us to take into account the causal role of both lumbar puncture and steroids in the pathogenesis of CVT. Lumbar puncture, especially following spinal and epidural anaesthesia or myelography is a rare, but well documented cause of CVT [5]. The exact mechanism underlying CVT occurrence after lumbar puncture is unknown. We hypothesize that lumbar puncture is followed by CSF hypotension, venous dilatation and stasis. This may favor the occurrence of a thrombotic process. If the later develops, the decrease in CSF reabsorption induces an increase in CSF pressure. This shift from CSF hypotension to CSF hypertension justifies the sig- nificant change in the headache characteristics. Headache due to CSF hypotension is, in fact, intermittent, postural, orthostatic, and with greater intensity when the subject is sitting or standing, whereas it regresses when the patient is lying.

In contrast, headache due to CSF hypertension is usually persisting and often worsens when the subject lies down. A papilledema may be revealed during fundus oculi examination and uni- or bilateral paresis of the sixth cranial nerve may occur. The change in headache characteristics may lead us to suspect CVT. In particular, when a headache occurs after a lumbar puncture and loses its postural characteristics, becomes persisting and worsens, one should suspect CVT, or alternatively, other complications such as subdural hematoma or meningitis, even in the absence of other neurological symptoms. In these conditions specific instrumental examinations are mandatory for diagnosis.

A multifactorial genesis has been often attributed to CVT. In the other cases described in literature, this pathologic condition, following a lumbar puncture, was associated with concomitant conditions, such as contraceptive assumption, disorders of thrombophilic status and puerperium. Certain abnormalities in the coagulation inhibitory systems, involving protein $\mathrm{C}$, protein $\mathrm{S}$, antithrombin and Leiden factor $\mathrm{V}$, are inherited in an autosomal dominant fashion and could predispose to thrombotic events [6, 7]. These abnormalities are responsible for $25 \%$ of deep venous thromboses and should be systematically checked, particularly in young patients and in those with a personal history of thrombosis. In the present case report, the complete study of the thrombophilic status of our patient did not reveal any abnormalities.

The pathogenetic role of steroids at high dosages for treating multiple sclerosis relapses remains uncertain with respect to the occurrence of CVT. High steroid doses favor a hypercoagulability status, as observed, for example, in patients with Cushing's disease or after intrathecal corticosteroid infusion by the temporal sequence between steroid administration and occurrence of a thrombophilic condition [8].

Greater significance should be attributed to the clinical picture: the modification in the pattern of headache after lumbar puncture and the worsening of headache after the steroid cycle represent a warning sign which must be adequately assessed with the aim of excluding the occurrence of CVT. We suggest a careful clinical monitoring of headache characteristics and its eventual changes in patients affected by multiple sclerosis who undergo lumbar puncture and successful steroid treatment [4] to exclude complications such as CVT [9]. In particular, steroid treatment after a lumbar puncture should be performed with caution. 


\section{References}

1. Ameri A, Bousser MG (1992)

Cerebral venous thrombosis. Neurol Clin 10:11-87

2. Deschiens MA, Conard J, Horellon MM et al (1966) Coagulation studies factor V Leiden and cardiolipin antibodies in 40 cases of cerebral venous thrombosis. Stroke 27:1724-1730

3. Hartley RW, Jackson A, Cooke RS (1992) Dural venous sinus thrombosis following myelography: demonstrations by magnetic resonance imaging. Br J Radiol 65:1134-1136
4. Aidi S, Chaumu, Biousse V, Bousser MG (1999) Changing pattern of headache pointing to cerebral venous thrombosis after lumbar puncture and intravenous high-dose corticosteroids. Headache 39:559-564

5. Wilder-Smith E, Kothbauer-Margreiter I, Lammle B et al (1997) Dural puncture and activated protein $C$ resistence: risk factors for cerebral venous sinus thrombosis. J Neurol Neurosurg Psychiatry 63:351-356

6. Dahalback B (1995) Inherited thrombophilia: resistance to activated protein $\mathrm{C}$ as a pathogenic factor of venous thromboembolism. Blood 85: 607-614
7. Zuber M, Toulon P, Marnet L et al (1996) Factor V Leiden mutation in cerebral venous sinus thrombosis.

Stroke 27:1721-1723

8. Bousser MG, Ross Russel RR (1997) Cerebral venous thrombosis. WB Saunders, London

9. Albucher JF, Vuillemin-Azais C, Manelfe C, Clanet M, GuiraudChaumeil B, Chollet F (1999) Cerebral thrombophlebitis in three patients with probable multiple sclerosis. Cerebrovasc Dis 9:298-303 